\title{
Downregulation of microRNA-34 induces cell proliferation and invasion of human mesothelial cells
}

\author{
NORIMITSU TANAKA ${ }^{1}$, SHINICHI TOYOOKA ${ }^{1}$, JUNICHI SOH ${ }^{1}$, KAZUNORI TSUKUDA $^{1}$, \\ KAZUHIKO SHIEN $^{1}$, MASASHI FURUKAWA ${ }^{1}$, TAKAYUKI MURAOKA ${ }^{1}$, YUHO MAKI ${ }^{1}$, TSUYOSHI UENO ${ }^{1}$, \\ HIROMASA YAMAMOTO $^{1}$, HIROAKI ASANO ${ }^{1}$, TAKEMI OTSUKI ${ }^{2}$ and SHINICHIRO MIYOSHI ${ }^{1}$
}

${ }^{1}$ Department of Thoracic Surgery, Okayama University Graduate School of Medicine, Dentistry and Pharmaceutical Sciences, Okayama 700-8558; ${ }^{2}$ Department of Hygiene, Kawasaki Medical School, Kurashiki, Okayama 701-0192, Japan

Received October 24, 2012; Accepted December 14, 2012

DOI: $10.3892 / o r .2013 .2351$

\begin{abstract}
Malignant mesothelioma (MM) is an aggressive tumor with a dismal prognosis, and the molecular alterations involved in this disease remain unknown. We previously reported that microRNA-34s (miR-34s) are methylated and downregulated in MM and may play an important role in the carcinogenesis of MM. In this study, we downregulated miR-34s in human mesothelial cells to investigate the cellular effect of miR-34 knockdown. For the cell study, we used LP-9, a human mesothelial cell line, and three human primary-cultured mesothelial cell lines. RNA-based miR-34a, $-34 b$ and $-34 c$ inhibitors were transfected into these cells, and their effects on proliferation and invasion were evaluated. A scramble RNA oligonucleotide was used as a control. The protein expression status was estimated using western blotting. After miR-34 inhibitor transfection, miR-34a, $-34 b$ and $-34 \mathrm{c}$ were downregulated in all the examined mesothelial cell lines. miR-34 inhibitor transfection significantly increased cell proliferation in all of the mesothelial cell lines, compared with the scramble control. The invasive ability also increased in the miR-34 inhibitor transfectants, compared with the scramble control, in the LP-9 cell line. Western blotting confirmed the upregulation of c-MET, phospho-c-MET, and Bcl-2 proteins in LP-9 cells after miR-34 inhibitor transfection. In conclusion, our study showed that the downregulation of miR-34s induced an oncogenic phenotype in non-malignant mesothelial cells. The present study, together with the results of our previous report, strongly suggest that $\mathrm{miR}-34$ s play an important role in
\end{abstract}

Correspondence to: Dr Shinichi Toyooka, Department of General Thoracic Surgery, Okayama University Graduate School of Medicine, Dentistry and Pharmaceutical Sciences, 2-5-1 Shikata-cho, Kita-ku, Okayama 700-8558, Japan

E-mail: toyooka@md.okayama-u.ac.jp

Abbreviations: MM, malignant mesothelioma; miR, microRNA; HPMCs, human primary-cultured mesothelial cells

Key words: mesothelial cells, carcinogenesis, non-coding RNA, microRNA-34, mesothelioma the early carcinogenic process involved in the transformation of human mesothelial cells to MM.

\section{Introduction}

Malignant mesothelioma (MM) is an aggressive tumor with a poor prognosis that arises most commonly in the pleura or peritoneum $(1,2)$. MM was once a rare disease, but its incidence has increased worldwide, probably as a result of widespread exposure to asbestos (3). However, a standard curative modality, such as radiotherapy, conventional chemotherapy or molecular targeting therapy, has not yet been established for advanced MM. In addition, much less information regarding the molecular alterations involved in MM is available, compared with other solid neoplasms (4).

microRNAs (miRNAs) are a conserved class of noncoding 20-22 nt small RNAs that regulate protein expression by binding to mRNA, leading to mRNA degradation or the inhibition of translation (5-7). Among the miRNAs, the microRNA-34 (miR-34) family members are direct transcriptional targets of p53, constituting part of the p53 tumor-suppressive network and inducing cell cycle arrest, apoptosis and senescence, which are the major consequences of p53 activation $(8,9)$. We previously reported that miR-34b/c was frequently downregulated by aberrant methylation in $\mathrm{MM}$, resulting in the loss of tumor-suppressive p53 function and the acquisition of a malignant phenotype (10). One of the unique molecular features of MM is that mutations and deletions of the TP53 gene are rare (11), even though MM generally exhibits cell cycle alterations and anti-apoptosis, suggesting functional p53 deficiency (12).

In the present study, unlike our previous report, we downregulated miR-34s in human mesothelial cells to investigate the cellular biological effects of miR-34 inhibition in human mesothelial cells and to elucidate the cancer mechanisms involved in MM.

\section{Materials and methods}

Cell lines and cell culture. A human mesothelial cell line (LP-9, peritoneal mesothelial cells) and three types of human primary-cultured mesothelial cells (HPMCs) were used in 
this study. The LP-9 cell line was purchased from the Coriell Cell Repository (Camden, NJ), and the three HPMCs were established from pleural effusions obtained from cancer-free patients treated at the Okayama University Hospital (Okayama, Japan), as described in our previous report (13). Approval from the Institutional Review Board and informed consent from all the patients were obtained.

LP-9 and HPMCs were cultured using Ham's F12 medium/Medium 199 (1:1 mixture) with 15\% fetal bovine serum, $2 \mathrm{mM}$ L-glutamine, $1.7 \mathrm{nM}$ epidermal growth factor and $1100 \mathrm{nM}$ hydrocortisone. All the cells were incubated at $37^{\circ} \mathrm{C}$ in a humidified atmosphere with $5 \% \mathrm{CO}_{2}$.

Transfection of inhibitors of anti-miRNA-34s. LP-9 and the three HPMCs were transfected with a scramble control oligonucleotide or Anti-miR ${ }^{\mathrm{TM}} \mathrm{miR}-34 \mathrm{a},-34 \mathrm{~b}$ and $-34 \mathrm{c}$ inhibitors (Ambion, Austin, TX) after being seeded in 6-well plates. Each miRNA inhibitor (150 pmol) in $200 \mu \mathrm{l}$ of serum-free antibiotic-free medium was mixed with $5 \mu$ l of Lipofectamine 2000 transfection reagent (Invitrogen, Carlsbad, CA) dissolved in $200 \mu \mathrm{l}$ of the same medium and allowed to stand at room temperature for $20 \mathrm{~min}$. The resulting $400 \mu \mathrm{l}$ transfection solutions were then added to each well containing $1.6 \mathrm{ml}$ of medium supplemented with $15 \%$ FBS. Cells were grown and harvested $48 \mathrm{~h}$ after the transfection for additional analyses.

Expression of $\mathrm{miR}-34 \mathrm{~s}$ as determined using quantitative $R T-P C R$. The miRNA was isolated from LP-9 and mesothelial cells using the TaqMan MicroRNA Cells-to-CT ${ }^{\mathrm{TM}}$ kit (Ambion) and treated with DNase I (Ambion) to remove genomic DNA. A reverse transcriptional (RT) reaction was performed to extract $0.5 \mu \mathrm{g}$ of miRNA using the TaqMan MicroRNA Reverse Transcriptional Kit system (Applied Biosystems) and TaqMan single RT primers for each miRNA (Applied Biosystems). Quantitative RT-PCR for miR-34a, -34b and $-34 c$ was performed using TaqMan MicroRNA Assay technology (Perkin-Elmer Corp., Foster City, CA) with the StepOnePlus ${ }^{\mathrm{TM}}$ Real-Time PCR system (Applied Biosystems). miR-374 expression was used to normalize the expression of the miR-34s as an endogenous control for the cell lines, following the manufacturer's recommendation (www.appliedbiosystems.com).

MTS assay. Cells were plated in 96-well plates at a density of $2.0 \times 10^{3}$ cells/well. Cell viability was evaluated at 0 and 3 days using an MTS assay with CellTiter $96^{\circledR}$ AQueous One Solution reagent (Promega, Madison, WI).

Colony formation assay. The in vitro cell proliferation was assessed by liquid colony formation assay. Viable cells (100) were plated onto 6 -well plates in triplicate. Cells were cultured and counted 14 days later after staining with $0.1 \%$ crystal violet in $20 \%$ ethanol for $5 \mathrm{~min}$ at room temperature. The number of visible colonies ( $>50$ cells) was counted.

Immunohistochemistry for Ki-67. miR-34 inhibitor-transfected or scramble control-transfected LP-9 cells were grown and treated in Lab-Tek chamber slides (Nunc, Naperville, IL). Medium was aspirated and cells were fixed in $4 \%$ paraformaldehyde for $10 \mathrm{~min}$ at room temperature. Paraformaldehyde was aspirated, and the cells were treated with a $0.2 \%$ Tween 20 in PBS for 15 min. Cells were then washed in PBS, and Ki-67 (Novocastra, Newcastle, UK) was added at a dilution of 1:2,000 in $1 \%$ bovine serum albumin and incubation was carried out overnight at $4^{\circ} \mathrm{C}$. Cells were washed in PBS before incubating in the dark with an FITC-labeled secondary antibody (Jackson ImmunoResearch, West Grove, PA) at a dilution of 1:100 in 1\% bovine serum albumin for $1 \mathrm{~h}$. The secondary antibody solution was aspirated, and the cells were washed in PBS. Cells were incubated in the dark with 4',6-diamidino-2-phenylindole $(1 \mu \mathrm{g} / \mathrm{ml})$ in PBS for $30 \mathrm{~min}$ and washed, and coverslips were mounted with an anti-fade solution (Dako Corp., Carpinteria, CA). The Ki-67 staining was evaluated using labeling index. At least 1,000 cells were counted under a microscope at a magnification of $\mathrm{x} 100$.

Soft-agar colony formation assay. To investigate the anchorage-independent growth potential of miR-34 inhibition, we performed soft agar colony formation assay. Cells $(7,500)$ in growth medium containing $0.4 \%$ agarose were placed on a $60-\mathrm{mm}$ dish with a $0.5 \%$ agarose base. After 3 weeks of incubation, the colonies were stained with $0.005 \%$ crystal violet at room temperature for $1 \mathrm{~h}$ and were counted for each dish. A549 cells (human lung adenocarcinoma cell line) were used as a positive control.

Cell migration and invasion assays. Cell migration and invasion were assayed using a Boyden chamber assay with filter inserts (pore size, $8 \mu \mathrm{m}$ ) in 6-well dishes (BD Biosciences Discovery Labware, Bedford, MA). Tumor cells in $2 \mathrm{ml}$ of serum-free medium (300 $\mu \mathrm{l}$ containing $0.75 \times 10^{5}$ cells for the Transwell migration assay and $1.5 \times 10^{5}$ cells for the Matrigel invasion assay) were added to the top chamber. The bottom chamber was prepared with $15 \%$ FBS as a chemoattractant. After a 24- and 48-h incubation for the migration and invasion assays, respectively, the non-invasive cells were removed by scrubbing with a cotton swab. The cells that migrated through the membrane and adhered to the lower surface of the membrane were fixed and stained using Diff-Quik stain (Sysmex, Kobe, Japan). To quantify the migration and invasion, the cells were counted under a microscope in 5 predetermined fields at a magnification of x100 and were represented as the average of three independent experiments.

Flow cytometric analysis. Cells were harvested and resuspended in PBS containing $0.2 \%$ Triton X-100 and $1 \mathrm{mg} / \mathrm{ml}$ RNase for $5 \mathrm{~min}$ at room temperature and then stained with propidium iodide (PI) at $50 \mathrm{mg} / \mathrm{ml}$ to determine subdiploid DNA content using a FACScan. Doublets, cell debris and fixation artifacts were gated out, and cell cycle analysis was carried out using CellQuest version 3.3 software.

Western blot analysis. Cells were grown to $80 \%$ confluence and harvested in lysis buffer [ $20 \mathrm{mmol} / 1$ Tris- $\mathrm{HCl}(\mathrm{pH} 7.5)$, $150 \mathrm{mmol} / 1 \mathrm{NaCl}, 1 \mathrm{mmol} / 1 \mathrm{Na}_{2}$ EDTA, $1 \mathrm{mmol} / 1$ EGTA, $1 \%$ Triton, $2.5 \mathrm{mmol} / 1$ sodium pyrophosphate, $1 \mathrm{mmol} / 1$ $\beta$-glycerophosphate, $1 \mathrm{mmol} / 1 \mathrm{Na}_{3} \mathrm{VO}_{4}, 1 \mu \mathrm{g} / \mathrm{ml}$ leupeptin] (Cell Signaling Technology, Beverly, MA) supplemented with Complete Mini (Roche, Basel, Switzerland) to extract the proteins. A total of $20 \mu \mathrm{g}$ of protein was separated using 


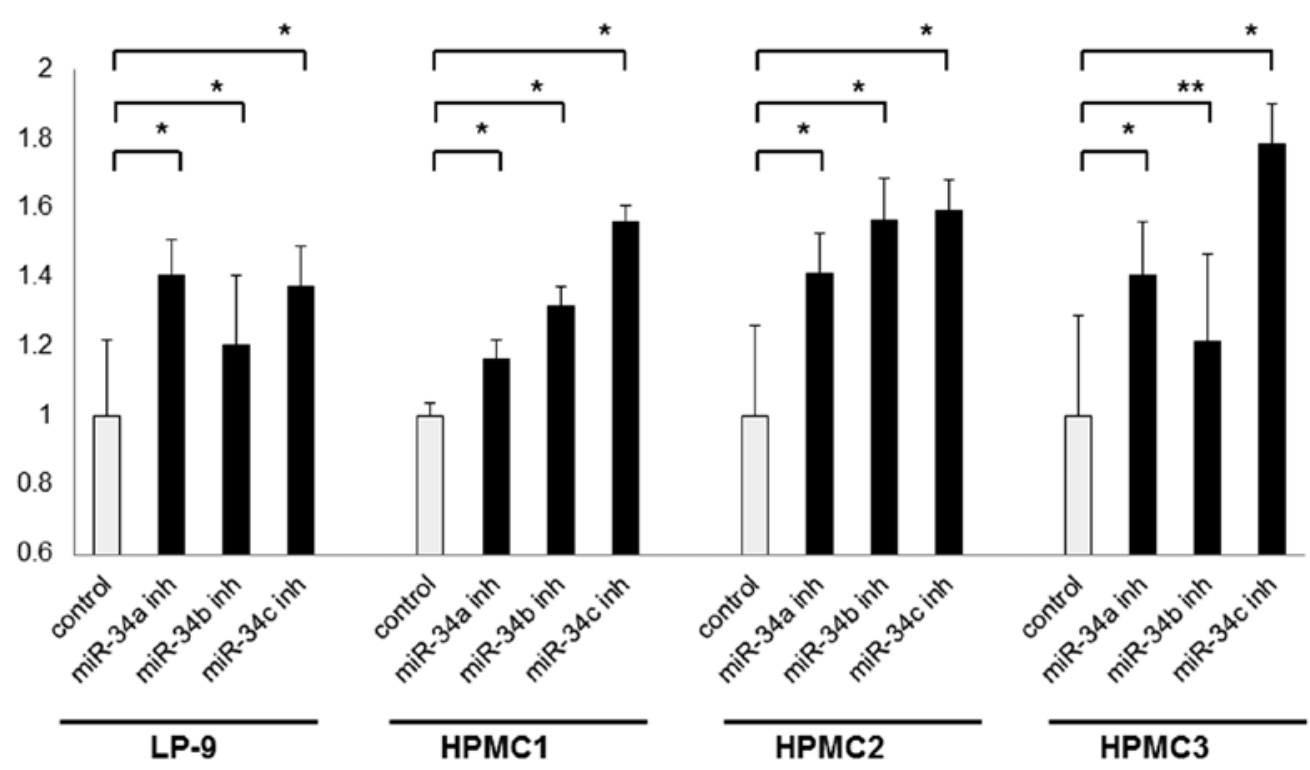

Figure 1. Effects of the inhibition of miR-34s on cell viability were evaluated using MTS assays. Control, scramble control; miR-34a, miR-34b and miR-34c inh indicate anti-miR-34a, anti-miR-34b and anti-miR-34c inhibitors. HPMC, human primary-cultured mesothelial cell. Values are expressed as the means \pm SD of three experiments. ${ }^{*} \mathrm{P}<0.01,{ }^{* *} \mathrm{P}=0.01$.

SDS-PAGE and was then transferred to PVDF membranes. The proteins on the membranes were incubated overnight at $4^{\circ} \mathrm{C}$ with the primary antibodies. The primary antibodies used for western blotting were as follows: anti-MET $(25 \mathrm{H} 2$; Cell Signaling), anti-phospho-MET (3D7, Tyr1234/1235; Cell Signaling) and anti-bcl-2 (human specific; Cell Signaling). The following secondary antibodies were used: goat anti-rabbit or anti-mouse IgG-conjugated horseradish peroxidase (Santa Cruz Biotechnology, Santa Cruz, CA). To detect the specific signals, the membranes were examined using ECL Plus Western Blotting Detection reagents (Amersham Biosciences UK Ltd., Buckinghamshire, UK).

Statistical analysis. The statistical analysis was performed using SPSS for Windows version 17.0 (SPSS Inc., Chicago, IL, USA). All of the in vitro experiments were performed at least three times. Data are represented as the means \pm standard deviation. The significance of the differences between the two groups was determined using the Chi-square test and the Mann-Whitney U-test, as appropriate. A 5\% significance level $(\mathrm{P}<0.05)$ was considered to indicate a statistically significant result.

\section{Results}

miR-34 inhibition by transfection with miR-34 inhibitors. We transfected the LP-9 cells and HPMCs with a scramble control and miR-34 inhibitors and confirmed that the expression of the miR-34s was suppressed in all of the cells, compared with the scramble control, using a real-time PCR method: 80-89\% inhibition for the miR-34a inhibitor, $45-73 \%$ for miR-34b and $68-70 \%$ for miR-34c.

Impact of miR-34 inhibitors on cell viability and proliferation. To screen for the cell viability effect of miR-34 inhibition, we performed MTS assays in LP-9 cells and the three mesothelial cell lines using transient transfection. miR-34a, $-34 \mathrm{~b}$ and $-34 \mathrm{c}$ inhibitors significantly increased the cell viability of all the examined cells, compared with the scramble control (1.2- to 1.4-fold for miR-34a, 1.2- to 1.6-fold for miR-34b, and 1.4- to 1.8-fold for miR-34c) (Fig. 1). In addition, to screen for the cell proliferation potential of miR-34 inhibition, we performed a colony formation assay and investigated the expression of Ki-67 in the LP-9 cells. The number of visible colonies was significantly increased in the cells transfected with the miR-34 inhibitors, compared with the scramble control (Fig. 2). The number of Ki-67-stained cells was significantly increased in cells transfected with the miR-34a $(\mathrm{P}<0.01)$ and $-34 \mathrm{c}(\mathrm{P}<0.01)$ inhibitors, compared with the scramble control. However, cells transfected with the miR-34b inhibitor tended to have increased numbers of Ki-67-stained cells ( $\mathrm{P}=0.09$ ) (Fig. 3). Regarding the anchorage-independent growth potential of miR-34 inhibition, LP-9 cells transfected with both the scramble control and miR-34 inhibitors did not grow in soft agar.

Impact of miR-34 inhibitors on migration and invasion. Cell migration and invasion potential were examined using a Boyden chamber. Microscopy images of the Boyden chamber assay are shown in Fig. 4. Both migration and invasion were significantly increased in the LP-9 cells transfected with all miR-34 inhibitors $(\mathrm{P}<0.01)$, compared with the scramble control. Parental HPMCs did not exhibit migration or invasion in this study, and such features were not acquired after transfection with miR-34 inhibitors.

Cell cycle analysis of LP-9 cells transfected with miR-34 inhibitors. Cell cycle analysis was conducted for LP-9 cells transfected with the scramble control or miR-34 inhibitors. The LP-9 cells transfected with the miR-34 inhibitors showed a slight decrease in the G0-G1 cell fraction, indicating that miR-34 inhibitors reduced G1 arrest (2.0-4.6\% decrease for miR inhibitors) (data not shown). 
A

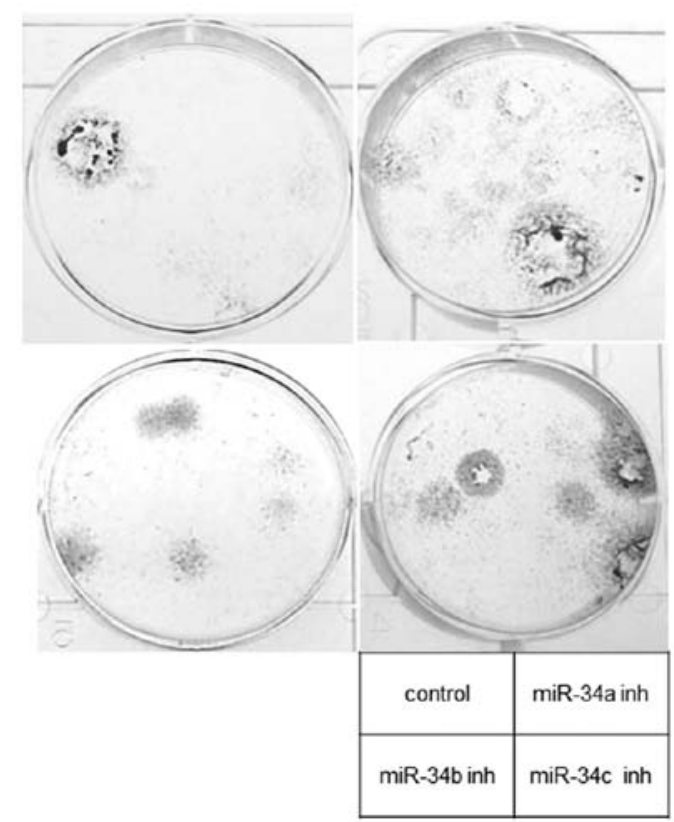

B

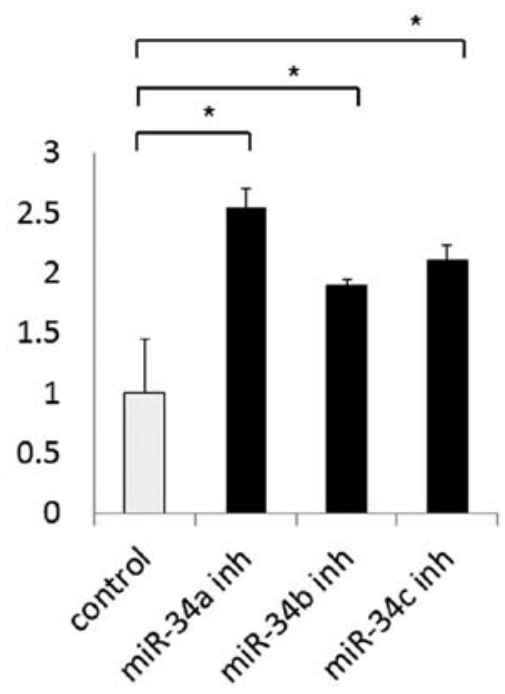

Figure 2. Effects of the inhibition of miR-34s on cell proliferation were evaluated using a colony formation assay in LP-9 cells. (A) Representative images of colony formation assay. (B) The number of colonies in control was set as 1 and the number of colonies in the treated cells was compared. Control, scramble control; miR-34a, miR-34b and miR-34c inh indicate anti-miR-34a, anti-miR-34b and anti-miR-34c inhibitors. Values are expressed as the means \pm SD of three experiments. $\mathrm{P}<0.01$.

A
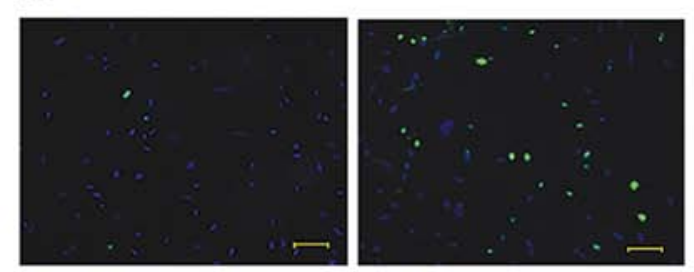

\begin{tabular}{|c|c|}
\hline control & $\begin{array}{c}\text { miR-34a } \\
\text { inh }\end{array}$ \\
\hline $\begin{array}{c}\text { miR-34b } \\
\text { inh }\end{array}$ & $\begin{array}{c}\text { miR-34c } \\
\text { inh }\end{array}$ \\
\hline
\end{tabular}

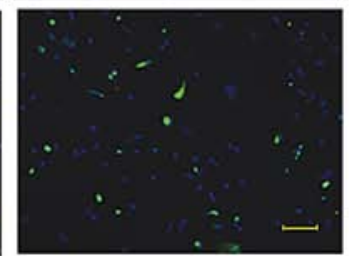

B

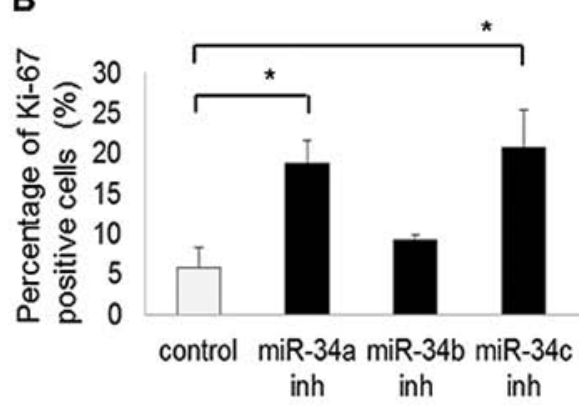

Figure 3. Effects of the inhibition of miR-34s on the immunofluorescent analysis of Ki-67 protein in LP-9 cells. (A) Representative images of immunofluorescent staining of Ki-67. Scale bars, $100 \mu \mathrm{m}$. (B) The percentage of Ki-67-positive cells in the treated cells was compared with that of the control. Control, scramble control; miR-34a, miR-34b and miR-34c inh indicate anti-miR-34a, anti-miR-34b and anti-miR-34c inhibitors. Values are expressed as the means \pm SD of three microscopic fields. ${ }^{*} \mathrm{P}<0.01$.

Protein expression of LP-9 cells transfected with miR-34 inhibitors. To examine the effect of miR-34 inhibition, we focused on c-MET (both total and phosphorylated types) and $\mathrm{Bcl}-2$, which are putative targets of miR-34s. Western blotting was performed using LP-9 cells transfected with the scramble control or miR-34 inhibitors. The total and phosphorylated c-MET and Bcl-2 expression levels were upregulated in the LP-9 cells transfected with the miR-34 inhibitors (Fig. 5).

\section{Discussion}

In the present study, we found that the downregulation of miR-34s in human mesothelial cells prompted increased cell viability, proliferation, resistance to apoptosis, and invasive potential but failed to increase anchorage-independent growth potential. We previously reported that the epigenetic silencing of miR-34b/c by methylation was extremely common $(100 \%$ of cell lines) and played an important role in the tumorigenesis of MM. In that study, miR-34a was also found to be downregulated by methylation (30\% of cell lines), suggesting a tumorigenic role in MM (10). Of note, Ji et al (14) reported that the restoration of miR-34s significantly inhibited clonogenic growth, while the inhibition of endogenous miR-34s by miR-34 inhibitors promoted growth in human pancreatic cancer cell lines. These results suggest that the inhibition of miR-34s is an important factor contributing to MM carcinogenesis.

Regarding the study of the oncogenic transformation of normal cells, the introduction of oncogenes, such as $K-R A S$ 
A

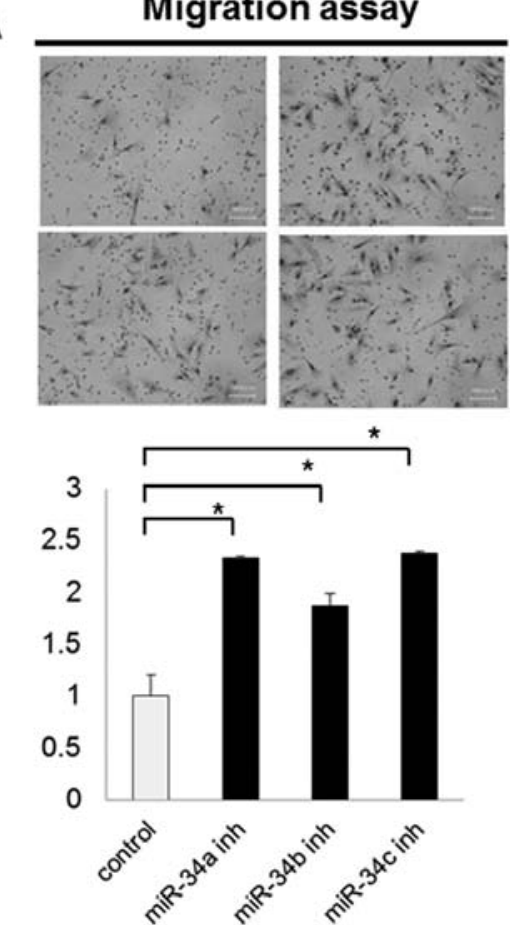

B

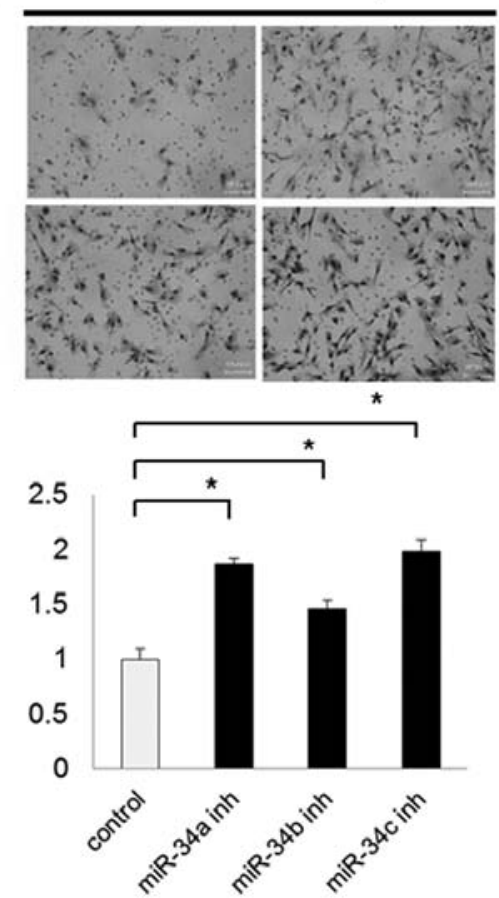

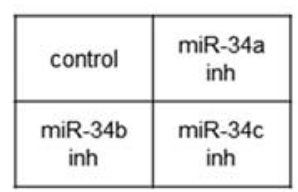

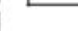

Figure 4. Effects of the inhibition of miR-34s on migration and invasive potential using migration (A) or invasion (B) assays in LP-9 cells. Control, scramble control; miR-34a, miR-34b and miR-34c inh indicate anti-miR-34a, anti-miR-34b and anti-miR-34c inhibitors. The quantitative values expressed as the means \pm SD of five microscopic fields are representative of three experiments. Scale bars, $100 \mu \mathrm{m}$. ${ }^{*} \mathrm{P}<0.01$.

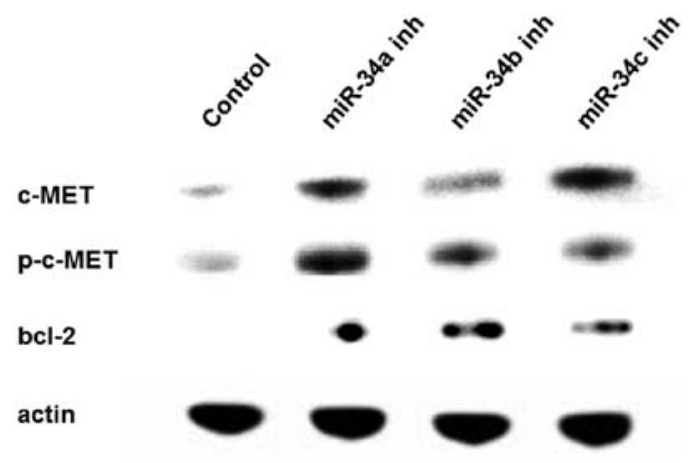

Figure 5. Effects of the inhibition of miR-34s on the protein expression profile of c-MET, p-c-MET and bcl-2 in LP-9 cells. Control, scramble control; miR-34a, miR-34b and miR-34c inh indicate anti-miR-34a, anti-miR-34b and anti-miR-34c inhibitors.

or HRAS and $c-M Y C$, caused the induction of a malignant phenotype in human bronchial epithelial cells (15-17). Sato et al (18) also reported that additional genetic changes, such as p53 knockdown, $K-R A S^{V I 2}$, and mutant epidermal growth factor receptor (EGFR), either alone or in combination, caused the progression of human bronchial epithelial cells at least partly toward malignancy, including the development of characteristics such as a higher saturation density, anchorage-independent growth, and an invasive phenotype, but failed to induce tumor formation. In our study, miR-34 inhibition induced increased cell viability in all the human mesothelial cell lines, and increased cell proliferation and invasive potential in LP-9 cells. However, miR-34 inhibition failed to promote anchorage-independent growth potential, suggesting that the inhibition of miR-34s was not only sufficient to cause crude mesothelial cells to undergo apparent malignant transformation but that other molecular alterations are required for the carcinogenic process in human mesothelial cells. Analysis of the immunofluorescent staining of Ki-67 demonstrated that cells transfected with miR-34a and $-34 \mathrm{c}$ inhibitors exhibited significantly increased numbers of Ki-67-positive stained cells, compared with the scramble control, although the cells transfected with the miR-34b inhibitor did not. The absence of significant differences in the miR-34b inhibitor suggest that the inhibition efficiency of the miR-34b inhibitor was lower than that of the miR-34a and $34 \mathrm{c}$ inhibitors in this study.

Several genes have been identified as targets of the miR-34s (9). In this study, $c-M E T$ and $B c l-2$ genes were examined as these gene products are considered to be important molecules in MM and were upregulated after miR-34 inhibition. c-MET was found to be activated in MM through overexpression or mutation, and its ligand, hepatocyte growth factor, was found to be overexpressed in MMs (19). Indeed, the suppression of c-MET using MET inhibitors revealed a potent inhibition of proliferation, invasion and migration in several MM cell lines (20). Bcl-2 is an anti-apoptotic protein located downstream of p53. The overexpression of Bcl-2 in MM has been reported in immunohistochemical analysis $(21,22)$ and is considered to be responsible for the anti-apoptotic feature of MM. Considering the results of western blot analysis together with colony formation assay and cell cycle analysis, our findings suggest that the inhibition of miR-34s increases the grade of malignancy in mesothelial cells through c-MET and Bcl-2. 
In conclusion, the present study, together with the findings of our previous report, strongly suggest that miR-34s play an important role in the early carcinogenic progression of human mesothelial cells to malignant mesothelioma.

\section{Acknowledgements}

We thank the Central Research Laboratory of the Okayama University Medical School for the technical support for the immunohistochemical staining. This study was supported by Grant-in-Aids for Scientific Research from the Ministry of Education, Culture, Sports, Science and Technology of Japan 22591566 (S.T.).

\section{References}

1. Robinson BW and Lake RA: Advances in malignant mesothelioma. N Engl J Med 353: 1591-1603, 2005.

2. Jakobsen JN and Sorensen JB: Review on clinical trials of targeted treatments in malignant mesothelioma. Cancer Chemother Pharmacol 68: 1-15, 2011.

3. Hansen J, de Klerk NH, Musk AW and Hobbs MS: Environmental exposure to crocidolite and mesothelioma: exposure-response relationships. Am J Respir Crit Care Med 157: 69-75, 1998.

4. Toyooka S, Kishimoto T and Date H: Advances in the molecular biology of malignant mesothelioma. Acta Med Okayama 62: 1-7, 2008.

5. Croce $\mathrm{CM}$ and Calin GA: miRNAs, cancer, and stem cell division. Cell 122: 6-7, 2005.

6. Hatfield S and Ruohola-Baker H: microRNA and stem cell function. Cell Tissue Res 331: 57-66, 2008

7. Zhang W, Dahlberg JE and Tam W: MicroRNAs in tumorigenesis: a primer. Am J Pathol 171: 728-738, 2007.

8. He L, He X, Lim LP, et al: A microRNA component of the p53 tumour suppressor network. Nature 447: 1130-1134, 2007.

9. Hermeking H: p53 enters the microRNA world. Cancer Cell 12: 414-418, 2007.

10. Kubo T, Toyooka S, Tsukuda K, et al: Epigenetic silencing of microRNA-34b/c plays an important role in the pathogenesis of malignant pleural mesothelioma. Clin Cancer Res 17: 4965-4974, 2011 .
11. Carbone M, Rizzo P, Grimley PM, et al: Simian virus-40 large-T antigen binds p53 in human mesotheliomas. Nat Med 3: 908-912, 1997.

12. Shimamura A and Fisher DE: p53 in life and death. Clin Cancer Res 2: 435-440, 1996.

13. Toyooka S, Pass HI, Shivapurkar N, et al: Aberrant methylation and simian virus 40 tag sequences in malignant mesothelioma. Cancer Res 61: 5727-5730, 2001.

14. Ji Q, Hao X, Zhang M, et al: MicroRNA miR-34 inhibits human pancreatic cancer tumor-initiating cells. PLoS One 4: e6816, 2009.

15. Reddel RR, Ke Y, Kaighn ME, et al: Human bronchial epithelial cells neoplastically transformed by v-Ki-ras: altered response to inducers of terminal squamous differentiation. Oncogene Res 3: 401-408, 1988.

16. Ura H, Bonfil RD, Reich R, et al: Expression of type IV collagenase and procollagen genes and its correlation with the tumorigenic, invasive, and metastatic abilities of oncogenetransformed human bronchial epithelial cells. Cancer Res 49: 4615-4621, 1989.

17. Yoakum GH, Lechner JF, Gabrielson EW, et al: Transformation of human bronchial epithelial cells transfected by Harvey ras oncogene. Science 227: 1174-1179, 1985.

18. Sato M, Vaughan MB, Girard L, et al: Multiple oncogenic changes (K-RAS(V12), p53 knockdown, mutant EGFRs, p16 bypass, telomerase) are not sufficient to confer a full malignant phenotype on human bronchial epithelial cells. Cancer Res 66: 2116-2128, 2006

19. Harvey P, Warn A, Newman P, Perry LJ, Ball RY and Warn RM: Immunoreactivity for hepatocyte growth factor/scatter factor and its receptor, met, in human lung carcinomas and malignant mesotheliomas. J Pathol 180: 389-394, 1996.

20. Jagadeeswaran R, Ma PC, Seiwert TY, et al: Functional analysis of c-Met/hepatocyte growth factor pathway in malignant pleural mesothelioma. Cancer Res 66: 352-361, 2006.

21. Soini Y, Kinnula V, Kaarteenaho-Wiik R, Kurttila E, Linnainmaa K and Paakko P: Apoptosis and expression of apoptosis regulating proteins bcl-2, mcl-1, bcl-X, and bax in malignant mesothelioma. Clin Cancer Res 5: 3508-3515, 1999.

22. O'Kane SL, Pound RJ, Campbell A, Chaudhuri N, Lind MJ and Cawkwell L: Expression of Bcl-2 family members in malignant pleural mesothelioma. Acta Oncol 45: 449-453, 2006. 